\title{
IL-19 Promotes Nasal Mucosal Tissue Remodeling in Chronic Rhinosinusitis Patients By Upregulating Fibronectin and Collagen I Expressions in Fibroblasts Via NF-kB-Smad2/3 Signaling Pathways
}

\section{Hongwei Bao}

Third Affiliated Hospital of Sun Yat-Sen University

Xia Li

Third Affiliated Hospital of Sun Yat-Sen University

Xiaoping Lai

Third Affiliated Hospital of Sun Yat-Sen University

Xiaoping Chen

Third Affiliated Hospital of Sun Yat-Sen University

Yue Li

Third Affiliated Hospital of Sun Yat-Sen University

Zhouzhou Yao

Third Affiliated Hospital of Sun Yat-Sen University

\section{Zizhen Huang}

Third Affiliated Hospital of Sun Yat-Sen University

Jiancong Huang

Third Affiliated Hospital of Sun Yat-Sen University

\section{Lihong Chang}

Third Affiliated Hospital of Sun Yat-Sen University

Gehua Zhang ( $\boldsymbol{\sigma}$ zhanggeh@mail.sysu.edu.cn )

Third Affiliated Hospital of Sun Yat-Sen University https://orcid.org/0000-0002-1917-0283

\section{Research}

Keywords: Chronic rhinosinusitis, fibroblast, IL-19, fibronectin, Collagen I, tissue remodeling

Posted Date: February 2nd, 2021

DOl: https://doi.org/10.21203/rs.3.rs-165739/v1

License: (c) (i) This work is licensed under a Creative Commons Attribution 4.0 International License.

Read Full License 
Page $2 / 20$ 


\section{Abstract}

\section{Background}

Chronic sinusitis without nasal polyps (CRSsNP), a subtype of chronic rhinosinusitis, is characterized by tissue remodeling, mainly by interstitial fibrosis. Fibroblasts are essential effectors of tissue remodeling, mainly expressing fibronectin (FN), collagen I (Col I), and other extracellular matrices. Our previous study found that IL-19 can promote the aberrant expression of extracellular matrix, and pre-experiments revealed that the Smad2/3 pathway plays a crucial role in tissue remodeling. However, the exact mechanism how IL-19 participants in tissue remodeling remains unclear. This study aimed to determine the roles of IL-19 in regulating the expression of FN and Col I, and the roles of Smad2/3 and NF-KB signaling pathways in fibroblasts.

\section{Methods}

Nasal mucosal tissue samples were collected from patients with chronic sinusitis with nasal polyps (CRSwNP), patients with CRSsNP, and controls to analyze the expression of IL-19, FN, and Col I by RTqPCR, immunohistochemistry, immunofluorescence, or western blotting. Cultured primary human nasal mucosal fibroblasts were treated with human recombinant IL-19 with or without Smad2/3 and NF-KB pathway inhibitor or agonist. They were analyzed for Smad2/3 and NF-KB pathway activation, and FN and Col I expression in fibroblasts by western blot, immunohistochemistry, and immunofluorescence.

\section{Results}

IL-19 co-localized with FN and Col I in nasal mucosal tissues and the expression levels of FN and Col I were elevated in CRSsNP, compared with CRSwNP and the control group. IL-19 activated the Smad2/3 and NF-KB pathways and promoted FN and Col I production in fibroblasts. NF-KB functions as an upstream pathway of the Smad2/3 pathway. The inhibitors of Smad2/3 and NF-KB could significantly attenuate the expression of FN and Col I induced by IL-19.

\section{Conclusion}

IL-19 promotes FN and Col I expression in fibroblasts via the NF-KB-Smad2/3 signaling pathway, leading to fibrosis and collagen deposition in patients with CRSsNP.

\section{Background}

Chronic rhinosinusitis (CRS) is a heterogeneous, chronic inflammatory disease of the nasal cavity and sinuses. Nearly $10 \%$ of the global population suffers from this disease, and its incidence is increasing annually [1]. In China, the prevalence of this disease is approximately $8 \%$ [2].

Tissue remodeling is a prominent pathological feature of chronic inflammation of the upper and lower airways and is a crucial determinant of the nature and prognosis of CRS. Chronic rhinosinusitis with 
nasal polyps (CRSwNP) is characterized by interstitial edema, accompanied by albumin deposition and decreased collagen synthesis in the extracellular matrix, which eventually leads to polyp formation. On the other hand, chronic sinusitis without nasal polyps (CRSsNP) is characterized by interstitial fibrosis and massive deposition of the extracellular matrix, including collagen, basement membrane thickening, and Goblet cell proliferation, accompanied by massive infiltration of neutrophils, which ultimately leads to mucosal thickening $[3,4]$. Fibroblasts play an essential role in maintaining tissue morphology and inducing extracellular matrix production. However, its overexpression leads to increased deposition of extracellular matrix components, such as fibronectin (FN) and collagen I (Col I), thus promoting fibrosis.

TGF- $\beta$ plays a central role in forming chronic fibrosis by regulating the function of fibroblasts, inducing fibroblasts to produce extracellular matrix, and promoting the accumulation of extracellular matrix $[5,6]$. Meanwhile, TGF- $\beta$, as an essential switch, plays a crucial role in the fibrosis process of the CRS subtype. It is precisely regulated during tissue remodeling by secreting various activating and inhibiting factors, such as LAP, LTBP, FN, integrin, and protease $[7,8]$.Smad2/3 protein is a substrate for type 1 TGF- $\beta$ receptors and mediates intracellular TGF- $\beta$ signaling $[9,10]$. It has been demonstrated that Smad proteins participate in tissue remodeling by activating TGF- $\beta[11,12]$. Studies have confirmed that blocking the TGF- $\beta$-mediated Smad2/3 classic pathway can reduce the deposition of nasal submucosal collagen, thereby inhibiting the fibrosis progression of chronic sinusitis tissues $[13,14]$. The NF-KB pathway is another signaling pathway that can influence fibroblast differentiation and significantly affects the expression of tissue remodeling associated proteins $[15,16]$.

The IL-20 subfamily, an essential class of pre-inflammatory factors, is considered to participate in tissue remodeling regulation by regulating extracellular matrix homeostasis, mucin deposition, and inflammatory cell infiltration in liver fibrosis, psoriasis, and other diseases [17-19]. Studies have found that IL-22 and IL-24, members of the IL-20 subfamily, play an essential role in liver fibrosis by enhancing TGF- $\beta$ activation signals and increasing the liver susceptibility to tumor inflammation [20].IL-20 levels were much higher in hepatocytes and hepatic stellate cells (HSCs) in liver biopsies of patients with fibrosis, cirrhosis, and liver cancer. It can also increase the expression of TGF, TNF, and Col1 and promotes the proliferation and migration of activated HSCs [21]. In psoriasis, IL-20 stimulates monocytes to produce pro-inflammatory cytokines and indirectly exerts its effect on keratinocyte proliferation through immune cells in the skin [22].In renal fibrosis disease, the pro-fibrotic effects of IL-19, IL-22, and IL-24 on the kidney was confirmed in a mouse renal disease model [23]. As a crucial member of the IL-20 subfamily, IL-19 has been found to be highly expressed in many chronic inflammatory diseases, such as psoriasis and asthma. It has been reported that LPS stimulation induces the upregulation of IL-19 and further increases the proliferation of nasal epithelial cells. Our previous study found higher expression of IL-19 and its receptors in nasal mucosal epithelial cells of CRS patients, and it promotes matrix metalloproteinase (MMP) expression, which plays critical roles in extracellular matrix balance [24-26]. However, whether a higher level of IL-19 plays a vital role in the fibrosis of CRS and the exact mechanism remains unclear. 
This study aimed to determine the correlation between IL-19 upregulation and nasal mucosal interstitial fibrosis in CRS patients and to investigate the effect and mechanism of IL-19 in regulating the expression of FN and Col I via the NF-KB and Smad2/3 signaling pathways.

\section{Materials And Methods}

\section{Patient and tissue samples}

CRS patients were diagnosed and classified, according to EPOS2012 [27]. Nasal polyps of CRSwNP patients, sinus mucosa of CRSsNP patients, and inferior turbinate tissue of control patients without sinusitis were collected. A portion of the specimens was stored at $-80^{\circ} \mathrm{C}$ and reserved in biobank of clinical resources of the third affiliated hospital of Sun Yat-sen university for immunohistochemistry and immunofluorescence testing of frozen sections. The remaining fresh specimens were soaked in PBS containing $1 \%$ antibiotic-antimicrobial agent and used for fibroblast cell culture. This study was approved by the ethics committee of the Third Affiliated Hospital of Sun Yat-sen University (NO.[2016]2-16). All subjects were included in the study only after informed consent was obtained.

\section{Human nasal mucosa primary fibroblast cell culture}

Human nasal mucosal tissues were cut into $1 \times 1$-mm fragments and digested with dispase II (50 $\mathrm{mg} / \mathrm{mL}$, Sigma-Aldrich, St. Louis, USA) overnight at $4{ }^{\circ} \mathrm{C}$ and further digested with trypsin at $37^{\circ} \mathrm{C}$ for 15 min. DMEM containing $10 \%$ fetal bovine serum was added to stop the digestion. The digested nasal tissues were then filtered through a 70- $\mu \mathrm{m}$ cell filter to obtain a cell suspension.

\section{Real-time quantitative PCR (RT-qPCR)}

RT-qPCR was used to detect the mRNA levels of the target genes coding for FN and Col I in the Control, CRSwNP, and CRSsNP groups, as previously described. Total RNA was extracted from cells using RNAiso Plus and was reverse transcribed to cDNA using the PrimeScript RT kit (TaKaRa Biotechnology). Realtime quantitative PCR was conducted using SYBR premix Ex Taq kit (TaKaRa Biotechnology) and the corresponding product primers (Invitrogen, Carlsbad, CA, USA). $\beta 2$ microglobulin ( $\beta 2 \mathrm{M}$ ) was used as a housekeeping gene for normalization. Relevant gene expression was analyzed using the comparative CT value method.

\section{Western Blot}

Proteins were extracted from cultured fibroblasts using a loading buffer (Thermo Scientific Inc., New York, USA) and boiled for $10 \mathrm{~min}$, electrophoresed on sodium dodecyl sulfate-polyacrylamide gel electrophoresis (SDS-PAGE, Sigma-Aldrich) and finally transferred to polyvinylidene difluoride membranes (PVDF) membranes. The membranes were blocked with $5 \%$ bovine albumin V (Biotop Technologies, Beijing, China) in tris-buffered saline (Boster Biotechnology, Wuhan) with tween-20 (TBST) (Solarbio Technologies, Beijing, China) for $2 \mathrm{~h}$ at room temperature and were incubated with primary anti-FN (1:1000, Abcam, Cambridge, UK), Col I (1:1000; Abcam, Cambridge, UK), p-Smad 2/3, Smad 2/3, plkBa, 
IKBa (1:1000; Cell Signaling Technology, Boston, USA) and GAPDH antibodies (1:3000; PeproTech, Rocky Hill, USA) overnight at $4{ }^{\circ} \mathrm{C}$. After washing in TBST buffer three times, the membranes were incubated with HRP-labeled secondary antibodies (Bioworld, Nanjing, China) at room temperature for $1 \mathrm{~h}$. The target proteins were visualized using the ECL reaction (Advansta, California, USA) with the FluorChem M System (protein simple, Alpha Innotech Corp). Image $\mathrm{J}$ software was used to analyze and quantify the density of the bands.

\section{Immunohistochemistry}

Tissues of surgical specimens from control, CRSwNP, and CRSsNP patients were fixed with $4 \%$ paraformaldehyde, paraffin-embedded, and cut into $4 \mu \mathrm{m}$-thick sections. The sections were dewaxed, rehydrated, and subjected to heat retrieval following routine procedures. The sections were covered with $10 \%$ goat serum (Boster Biotechnology) to block non-specific binding for $30 \mathrm{~min}$ and then incubated with anti- wave protein (Vimentin, VIM, 1:200, Immunoway, Company of Origin), FN and Col I antibodies (1:200, Cell Signaling Technology, Boston, USA) overnight at $4^{\circ} \mathrm{C}$. After washing three times with PBS, sections were incubated with HRP-labeled secondary antibodies (Bioworld, Nanjing, China) and detected under a 400x magnification microscope (Leica, Wetzlar, Germany). The results were expressed as staining intensity and the percentage of IHC-positive cells as percentages of the total epithelial area.

\section{Immunofluorescence staining and confocal microscopy}

For human nasal tissues, frozen slice sections of the tissues were immersed in acetone for $10 \mathrm{~min}$, allowed to warm up to room temperature, and then washed with TBS for $20 \mathrm{~min}$ on a shaker. After blocking with $5 \%$ albumin bovine V (Biotopped Science \& Technology, Beijing, China) in TBST $(0.1 \%$ Tween 20) for $1 \mathrm{~h}$, the sections were incubated with anti-FN or Col I antibody (1:200; Cell Signaling Technology, Boston, USA) overnight at $4{ }^{\circ} \mathrm{C}$ after blocking with $10 \%$ goat serum blocked for 30 min. For fibroblasts, cells were fixed with $4 \%$ paraformaldehyde, closed with $1 \%$ BSA for $30 \mathrm{~min}$, and then incubated with anti-FN, Col I, or p65 antibody (1:500; Cell Signaling Technology, Boston, USA) at $4{ }^{\circ} \mathrm{C}$ overnight. Subsequently, frozen tissue sections and fibroblasts were incubated with Alexa Fluor 594 -/- or 488-labeled secondary antibodies (1:1000; (Invitrogen, CA, USA) for 1 hour at room temperature. Finally, nuclei were labeled with 1:30,000 DAPI staining; Biolegend, CA, USA) and sealed with an anti-fade protectant (Invitrogen, CA, USA). Immunofluorescence was detected under a 400x magnification microscope (Leica, Wetzlar, Germany) or a 630x magnification confocal microscope (Carl Zeiss, Jena, Germany).

\section{Statistical analysis}

Statistical analysis was performed using IBM SPSS 20 (SPSS, Chicago, IL, USA). Three or more groups were analyzed for significance using one-way analysis of variance (ANOVA) or the Kruskal-Wallis test for comparative study. The $t$-test or Mann-Whitney U-test (2-tailed) was applied for comparison between groups. The paired $t$-test or Wilcoxon's paired with the signed-rank test was used to compare the two groups. Significance was set at $P<0.05$. 


\section{Results}

\section{Fibroblasts, FN and Col I, were highly expressed in CRSsNP tissues}

Immunohistochemistry showed the number and location of vimentin (VIM), suggesting that fibroblasts were mainly located in the basement membrane (located between the epithelial and lamina propria, as shown by the arrows in Figure 1). The expression of VIM in the CRSsNP group was significantly higher than that in the control and CRSwNP groups $(P<0.05$ for both, Figure $1 \mathrm{~A}, 1 \mathrm{~B})$. Both CRSwNP and CRSsNP demonstrated higher expression of FN and Col I when compared with the control group $(P<0.05$ for both, Figure 1A, 1B). Moreover, the CRSsNP patients showed the highest levels of FN and Col I in CRSsNP patients than in CRSwNP patients $(P<0.05$, Figure 1A, 1B).

\section{Co-localization of IL-19 with FN and Col I in mucosal tissues of CRSsNP patients}

Immunofluorescence showed that IL-19 co-localized with FN and Col I in the cytoplasm of the nasal mucosal epithelium. The expressions of FN and Col I in the CRSsNP group were higher than those in the control and CRSwNP groups (Figure 2).

\section{IL-19 promoted the expressions of FN and Col I in fibroblasts}

To further confirm the relationships between IL-19, FN, and Col I in fibroblasts, primary fibroblasts were cultured in vitro and pretreated with human recombinant IL-19 (hIL-19) or not. The protein levels of FN and Col I in fibroblasts were detected by western blotting after pretreatment with different concentrations of IL-19 for 24 hours. As shown in Figure 3A, the expression of FN and Col was significantly increased in fibroblasts treated with hIL-19-treated compared to the control. The optimum concentration of hIL-19 was $200 \mathrm{ng} / \mathrm{mL}$ ( $P=0.01$, for both). We further verified the immunofluorescence results, which also showed that the expression of FN and Col I was increased in the IL-19-treated group (Figure 3B). Furthermore, RTqPCR analysis showed that IL-19 promoted the mRNA expression of $\mathrm{FN}$ and Col I in fibroblasts at an optimum concentration of $200 \mathrm{ng} / \mathrm{mL}$ ( $P=0.001$ for $\mathrm{FN}, P=0.01$ for Col I, Figure $3 \mathrm{C}$ ). These results suggest that IL-19 promotes the expression of FN and Col I in fibroblasts at both the protein and transcript levels.

\section{IL-19 promoted FN and Col I expressions in fibroblasts via Smad2/3 pathway}

The role of TGF-induced fibrosis has been widely recognized, and Smad2/3, as a switch of TGF signaling, also plays an essential role in the development of tissue fibrosis [11, 12]. Therefore, to further explore the mechanism by which IL-19 promotes the expression of FN and Col I in fibroblasts and to verify the role of the Smad2/3 pathway further, we treated primary fibroblasts with IL-19 stimulation combined with Smad2/3 pathway inhibitor. It was found that hIL-19 could promote $\mathrm{p}-\mathrm{Smad} 2 / 3$ and Smad2/3 protein expression levels (Figure 4A). Furthermore, western blotting demonstrated that hIL-19 induced upregulation of p-Smad, FN and Col I were eliminated by the Smad pathway inhibitor (Indoxinmod) pretreatment (Fig. 4B). This was further confirmed by immunofluorescence (Fig. 4C). The results indicated that IL-19 promoted FN and Col I expression in fibroblasts through the Smad2/3 pathway. 


\section{IL-19 promotes FN and Col I expression in fibroblasts via NF-KB-Smad2/3 pathway}

Studies have reported that the NF-KB pathway is a class of classical pathways associated with inflammation in human liver and lung fibroblasts [28]. To further verify the NF-KB pathway's role, primary fibroblasts were treated with different doses of hIL-19, western blot analysis showed that IL-19 could promote $\mathrm{p}-\mathrm{IkBa}$ protein expression (Figure $5 \mathrm{~A}$ ). Furthermore, we treated fibroblasts with hIL-19 combined with NF-KB pathway inhibitors (Bay11-7082) and found that the FN and Col I protein expression in the IL19 combined with Bay11-7082 treatment group was lower than that in the IL-19 treatment alone $(P=0.01$, Fig. 5B). It was further confirmed by immunofluorescence that both NF-KB activator (EVP4953) and hIL19 treatment could increase the FN and Col I expression in fibroblasts, while the Bay combined with hIL19 treatment decreased FN and Col I expression (Figure 5C). The results indicated that IL-19 promoted FN and Col I expression in fibroblasts through the NF-kB pathway.

We further confirmed the upstream and downstream relationships between the NF-KB and Smad2/3 pathways. As shown in Figure 5D, after adding the Smad2/3 activator, the expression of $\mathrm{FN}$, Col I, and Smad2/3 increased significantly, but the increase in p-IкBa was not significant. After adding Smad2/3 inhibitor, the expression of Smad2/3 protein and FN, Col-1 decreased significantly, and the expression of $\mathrm{p}-\mathrm{IKBa}$ did not change much compared with the control group. NF-KB agonist treatment significantly increased the expression of $\mathrm{FN}, \mathrm{Col} \mathrm{I}$, and p-Smad2/3 protein significantly. The expression of FN, Col I, and $\mathrm{p}-\mathrm{Smad} 2 / 3$ protein decreased significantly after the NF-KB inhibitor's addition compared with the IL19 stimulation group. This was corroborated by the confocal assay and microscopic observation of FN, Col I and p-Smad2/3, and p-65 expression (Figure 5e). It is inferred that NF-KB may act as an upstream pathway of the Smad2/3 pathway and act as a bridge in the process of tissue fibrosis.

\section{Discussion}

Tissue remodeling is the main pathological feature of chronic inflammation of the upper and lower airways and is an essential factor in determining the nature and prognosis of CRS lesions [4]. In CRSwNP, extracellular connective tissue matrix degradation, markedly less collagen deposition might be a critical pathological step and feature, while, in CRSsNP, basement membrane thickening (fibrosis), collagen deposition, and goblet cell hyperplasia are the main characteristics, with extracellular matrix composition $[29,30]$.

Extracellular matrices (ECMs) are vital external environments that regulate cell and tissue homeostasis and play a critical role in tissue remodeling, a class of complex network structures consisting of a large variety of matrix macromolecules whose precise composition and specific structures vary from tissue to tissue. [31]. In the heart, muscle cells can produce collagen 4, 6, laminin (LN), and proteoglycans. Endothelial cells can produce collagen I, III,IV, LN, and fibronectin (FN), while fibroblasts can produce Col I, III, and FN to form ECMs [32,33]. It has been reported that in CRSwNP tissues, the major components of ECMs are collagen I, III, and FN; they are most highly expressed in non-eosinophilic CRSwNP. Collagen IV did not differ significantly among groups and was mainly distributed around blood vessels. In 
eosinophilic CRSwNP, the loss of matrix deposition may be due to decomposition or failure to produce the necessary components, with lower levels of extracellular matrix expression [34]. It was also reported that significant mucinous gland hyperplasia and collagen deposition were seen in the extracellular matrix of CRSsNP [35]. The cup cell number and basement membrane thickness of CRSsNP were significantly increased, and the collagen deposited in the ECMs consisted mainly of collagen I, with significantly less collagen III and IV [36]. Our experiments revealed that Col I and FN are highly expressed in the intrinsic layer of CRS nasal mucosal tissues and as their primary source of production from fibroblasts, and the expression of the fibroblast marker waveform protein was found to be increased in the CRSsNP patients. Also, the expression levels of FN and Col I were high in the mucosa of CRSsNP patients. Therefore, two proteins, Col I and FN, were selected as extracellular matrix markers for detecting different subgroups of chronic sinusitis. However, the mechanism underlying the higher FN and Col deposition in the mucosa of CRSsNP was unclear.

Our previous study reported that IL-19 was highly expressed in CRSsNP, especially in the epithelium of nasal mucosa, and correlated with MMPs, which are the primary regulators in manipulating ECM balance. It also contributes to mucus overproduction by promoting MUC5AC expression in CRS epithelial cells [25, 37]. This indicates that IL-19 plays a critical role in the tissue remodeling and pathological process of CRS. In this study, we found that FN and Col I co-localized with IL-19 in the nasal mucosa of CRS patients, and the expression levels of these two factors were found to be higher in the nasal mucosa of patients with CRSsNP than in those with CRSsNP. Moreover, stimulation of nasal fibroblasts with suitable concentrations of IL-19 promoted high expression of FN and Col I. This was consistent with the tissue remodeling characteristics that CRSsNP predominantly shows mesenchymal fibrosis, collagen deposition, and higher ECM composition, while CRSwNP presents edema and decreased ECM [29]. When stimulation with higher concentrations $(>200 \mathrm{ng} / \mathrm{mL}$ ) of hlL-19, the expression levels of FN and Col I in fibroblasts decreased, which was speculated to be related to the toxic effect of IL-19 on cells. Similar to the existing experiments, the study also confirmed that IL-24 in the IL-10 superfamily could be cytotoxic to tumor cells at a certain concentration level [38].

As an inhibitory effect on the extracellular matrix, IL-19 was reported to decrease the expression of TGF- $\beta$ and connective tissue growth factor (CTGF) in rat fibroblasts at day 7 , and further significantly inhibited the generation of extracellular a-SMA, Col-1, and FN at 28 days, via the Erk and p38 pathways [39]. In this study, IL-19 was found to activate the Smad2/3 pathway, a substrate for type 1 TGF- $\beta$ receptors, which plays a crucial role in transferring TGF- $\beta$ signals from cell surface receptors to the nucleus and mediates TGF- $\beta$ intracellular signaling. Currently, Smad proteins are divided into three categories based on their different functions in signal transduction in the TGF family: public mediator Smad proteins, inhibitory Smad proteins, and pathway-restricted Smad proteins, including Smad2 and Smad3. Activated TGF- $\beta$ receptor-induced pathway-restricted (specific) Smad 2 and Smad 3 undergo phosphorylation via cytosolspecific serine/threonine kinase receptor activation and bind to common Smad 4 for oligomeric translocation to the nucleus, where they directly respond to the transcriptional function of TGF- $\beta$. This structure serves as a switch for intracellular signaling by Smad. The role of protein-activated TGF in tissue remodeling fibrosis has also been demonstrated $[5,6,14,35]$. 
This study found that the NF-KB pathway can influence the IL-19 induced overproduction of FN and Col I in fibroblasts. Inhibition of NF-KB activity decreased the specificity of $\mathrm{p}-\mathrm{Smad} 2 / 3$ expression. Also, the present experiment, in which the expression of $\mathrm{FN}$ and Col I was detected by two-way validation by adding NF-KB and Smad2/3 pathway activators and inhibitors, confirmed that IL-19 promotes the production of extracellular matrix including $\mathrm{FN}$ and Col I through the NF-KB-Smad2/3 pathway. This was consistent with a previous study showing that TGF stimulated the expression of extracellular matrix including FN, Col I, and a-SMA in fibroblasts, decreased when combined with NF-KB pathway inhibitors $[16,40]$. This study also shows that the NF-KB pathway can influence the differentiation of myofibroblasts and significantly affect tissue remodeling and related protein expression.

In this study, we used IL-19 stimulation of fibroblasts to investigate the effects of FN and Col I upon activation of the NF-KB-Smad2/3 pathway. It was found that IL-19 affects the process of CRS tissue fibrosis by activating the NF-KB signaling pathway, which in turn activates the downstream Smad2/3 pathway and stimulates fibroblasts to produce Col and FN, leading to alterations in CRSsNP tissue remodeling. This study investigates the feasibility of preventing and reducing CRSsNP tissue fibrosis and provides potential therapeutic targets for the inhibition of pathological nasal tissue remodeling by investigating pathway activation and related protein expression in fibroblasts in CRSsNP. However, this study did not explore the specific process of Smad2/3 protein activation by IL-19 in detail and only demonstrated that IL-19 promotes the activation of the Smad2/3 pathway in fibroblasts, and the specific mechanism of Smad2/3 activation remains to be further explored.

\section{Declarations}

\section{Ethics approval and consent to participate}

The studies involving human participants were reviewed and approved by Third Affiliated Hospital, Sun Yat-sen University. The patients/participants provided their written informed consent to participate in this study.

\section{Consent for publication}

Consent to publish was obtained from all authors.

\section{Availability of data and materials}

All data in our study are available upon request.

\section{Competing interests}

There is no conflict of interest.

\section{Author Contributions}


Gehua Zhang conceived the study design, reviewed manuscript and provided comments. Lihong Chang supervised the experiments and data analysis, reviewed manuscript. Hongwei Bao drafted the manuscript and finished the majority of experiments, and acquired the data. Xia Li and Xiaoping Lai design experiments, data analysis and revised manuscript. Xiaohong Chen analyzed and interpreted the data, collected samples and revised manuscript. Zizhen Huang helped for the data analysis and recruited patients and collected samples. Yue Li and Zhouzhou Yao helped for the data analysis and sample collection. Jiancong Huang participant in the experiment design and supervision, and recruited patients and collected samples.

\section{Funding}

This study was supported by the National Natural Science Foundation of China $(81670913,81970859$ and 82071020), the Science and Technology Planning Project of Guangzhou, Guangdong Province (202002030034), Natural Science Foundation of Guangdong Province (2019A1515011093), and the Fundamental Research Funds of Central University (19ykpy30).

\section{Acknowledgments}

We thank for biobank of clinical resources of the third affiliated hospital of Sun Yat-sen university for the samples collection and conservation.

\section{References}

1. Wytske Fokkes, Valerie Lund, Isam Alobid, Wilma Terezinha AnselmoLima, Claus ns, Valerie Lund, Isam Alobid: European Position Paper on Rhinosinusitis and Nasal Polyps 2020. Rhinology 2020; 58: 1-464.

2. Shi JB, Fu QL, Zhang H, Cheng L, Wang YJ, Zhu DD, Lv W, Liu SX, Li PZ, Ou CQ, Xu G: Epidemiology of chronic rhinosinusitis: results from a cross-sectional survey in seven Chinese cities. Allergy 2015; 70:533-539.

3. Yan Y, Gordon WM, Wang DY: Nasal epithelial repair and remodeling in physical injury, infection, and inflammatory diseases. Curr Opin Otolaryngol Head Neck Surg 2013; 21:263-270.

4. Van Bruaene N, Bachert C: Tissue remodeling in chronic rhinosinusitis. Curr Opin Allergy Clin Immunol 2011; 11:8-11.

5. Caja L, Dituri F, Mancarella S, Caballero-Diaz D, Moustakas A, Giannelli G, Fabregat I: TGF-beta and the Tissue Microenvironment: Relevance in Fibrosis and Cancer. Int J Mol Sci 2018; 19(5): 1294.

6. Pardali E, Sanchez-Duffhues G, Gomez-Puerto MC, Ten Dijke P: TGF-beta-Induced EndothelialMesenchymal Transition in Fibrotic Diseases. Int J Mol Sci 2017; 18(10): 2157.

7. Min Y, Lee CH, Rhee C-S, Hong SK, Kwon SH: Increased Expression of IL-4,IL-5, IFN-gamma, IL-6, IL-8, and TGF-beta mRNAs in Maxillary Mucosa of Patients with Chronic Sinusitis. Am J Rhinol 1999; 13:339-343. 
8. Wang M, Ye T, Liang N, Huang Z, Cui S, Li Y, Huang Q, Zhou B: Differing roles for TGF-beta/Smad signaling in osteitis in chronic rhinosinusitis with and without nasal polyps. Am J Rhinol Allergy 2015; 29:e152-159.

9. Wrana JL: Crossing Smads. Sci STKE 2000; 2000 (23):re1.

10. Shi Y, Massagué J: Mechanisms of TGF- $\beta$ Signaling from Cell Membrane to the Nucleus. Cell 2003; 113:685-700.

11. Oh CJ, Kim JY, Min AK, Park KG, Harris RA, Kim HJ, Lee IK: Sulforaphane attenuates hepatic fibrosis via NF-E2-related factor 2-mediated inhibition of transforming growth factor-beta/Smad signaling. Free Radic Biol Med 2012; 52:671-682.

12. Akhurst RJ, Hata A: Targeting the TGFbeta signalling pathway in disease. Nat Rev Drug Discov 2012; 11:790-811.

13. Cutroneo KR: TGF-beta-induced fibrosis and SMAD signaling: oligo decoys as natural therapeutics for inhibition of tissue fibrosis and scarring. Wound Repair Regen 2007; 15 Suppl 1:S54-60.

14. Kim HJ, Park JH, Shin JM, Yang HW, Lee HM, Park IH: TGF-beta1-induced HSP47 regulates extracellular matrix accumulation via Smad2/3 signaling pathways in nasal fibroblasts. Sci Rep 2019; 9:15563.

15. Ichikawa T, Sugiura H, Koarai A, Kikuchi T, Hiramatsu M, Kawabata H, Akamatsu K, Hirano T, Nakanishi M, Matsunaga K, et al: 25-hydroxycholesterol promotes fibroblast-mediated tissue remodeling through NF-kappaB dependent pathway. Exp Cell Res 2013; 319:1176-1186.

16. Zhang Y, Jiao H, Wu Y, Sun X: P120-catenin regulates pulmonary fibrosis and TGF-beta induced lung fibroblast differentiation. Life Sci 2019; 230:35-44.

17. Chen J, Caspi RR, Chong WP: IL-20 receptor cytokines in autoimmune diseases. J Leukoc Biol 2018; 104:953-959.

18. Wegenka UM: IL-20: biological functions mediated through two types of receptor complexes. Cytokine Growth Factor Rev 2010; 21:353-363.

19. Caparros E, Frances R: The Interleukin-20 Cytokine Family in Liver Disease. Front Immunol 2018; 9:1155.

20. Rokonay R, Veres-Szekely A, Szebeni B, Pap D, Lippai R, Beres NJ, Veres G, Szabo AJ, Vannay A: Role of IL-24 in the mucosal remodeling of children with coeliac disease. J Transl Med 2020; 18:36.

21. Chiu YS, Wei CC, Lin YJ, Hsu YH, Chang MS: IL-20 and IL-20R1 antibodies protect against liver fibrosis. Hepatology 2014; 60:1003-1014.

22. Stenderup K, Rosada C, Worsaae A, Clausen JT, Norman Dam T: Interleukin-20 as a target in psoriasis treatment. Ann N Y Acad Sci 2007; 1110:368-381.

23. Pap D, Veres-Szekely A, Szebeni B, Rokonay R, Onody A, Lippai R, Takacs IM, Tisler A, Kardos M, Oswald F, et al: Characterization of IL-19, -20, and -24 in acute and chronic kidney diseases reveals a pro-fibrotic role of IL-24. J Transl Med 2020; 18:172. 
24. Sziksz E, Pap D, Lippai R, Beres NJ, Fekete A, Szabo AJ, Vannay A: Fibrosis Related Inflammatory Mediators: Role of the IL-10 Cytokine Family. Mediators Inflamm 2015; 2015:764641.

25. Lai X, Li X, Chang L, Chen X, Huang Z, Bao H, Huang J, Yang L, Wu X, Wang Z, et al: IL-19 UpRegulates Mucin 5AC Production in Patients With Chronic Rhinosinusitis via STAT3 Pathway. Front Immunol 2019; 10:1682.

26. Pace E, Scafidi V, Di Bona D, Siena L, Chiappara G, Ferraro M, La Grutta S, Gallina S, Speciale R, Ballacchino A, et al: Increased expression of IL-19 in the epithelium of patients with chronic rhinosinusitis and nasal polyps. Allergy 2012; 67:878-886.

27. Fokkens WJ, Lund VJ, Mullol J, Bachert C, Alobid I, Baroody F, Cohen N, Cervin A, Douglas R, Gevaert P, et al: EPOS 2012: European position paper on rhinosinusitis and nasal polyps 2012. A summary for otorhinolaryngologists. Rhinology 2012; 50:1-12.

28. Mitchell JP, Carmody RJ: NF-kappaB and the Transcriptional Control of Inflammation. Int Rev Cell Mol Biol 2018; 335:41-84.

29. Samitas K, Carter A, Kariyawasam HH, Xanthou G: Upper and lower airway remodelling mechanisms in asthma, allergic rhinitis and chronic rhinosinusitis: The one airway concept revisited. Allergy 2018; 73:993-1002.

30. Cho SH, Kim DW, Gevaert P: Chronic Rhinosinusitis without Nasal Polyps. J Allergy Clin Immunol Pract 2016; 4:575-582.

31. Theocharis AD, Skandalis SS, Gialeli C, Karamanos NK: Extracellular matrix structure. Adv Drug Deliv Rev 2016; 97:4-27.

32. Yamanaka Y, Gingery A, Oki G, Yang TH, Zhao C, Amadio PC: Blocking fibrotic signaling in fibroblasts from patients with carpal tunnel syndrome. J Cell Physiol 2018; 233:2067-2074.

33. Xing $\mathrm{H}$, Lee $\mathrm{H}$, Luo L, Kyriakides TR: Extracellular matrix-derived biomaterials in engineering cell function. Biotechnol Adv 2020; 42:107421.

34. Feng X, Payne SC, Borish L, Steinke JW: Differential Expression of Extracellular Matrix Components in Nasal Polyp Endotypes. Am J Rhinol Allergy 2019; 33:665-670.

35. Shieh JM, Tsai YJ, Chi JC, Wu WB: TGFbeta mediates collagen production in human CRSsNP nasal mucosa-derived fibroblasts through Smad2/3-dependent pathway and CTGF induction and secretion. J Cell Physiol 2019; 234:10489-10499.

36. Li XH, Zhan ZR, Sun JS, Zeng B, Xue YP, W SQ: Nasal mucosa remodeling in chronic rhinosinusitis without nasal polyps. Lin Chung Er Bi Yan Hou Tou Jing Wai Ke Za Zhi 2013; 27 (20): 1110-1113, 1117.

37. Li X, Chang LH, Huang ZZ, Wang ZY, Chen XZ, Wang J, Zhang GH: Correlation of IL-19 and its receptors with tissue remodeling in chronic rhinosinusitis. Chinese journal of pathophysiology 2017; 33 (5): 919-924.

38. Yu X, Xia W, Zhang T, Wang H, Xie Y, Yang J, Miao J: Enhanced cytotoxicity of IL-24 gene-modified dendritic cells co-cultured with cytokine-induced killer cells to hepatocellular carcinoma cells. Int J Hematol 2010; 92:276-282. 
39. Wang QJ, Zhang AL, Kang ZQ, Zhang ZT, Wang YS: Exogenous IL-19 mediates downregulation of TGF- $\beta$ through Erk and p38 pathway to inhibit epidural fibrosis. Eur Rev Med Pharmacol Sci 2019; 23 (17): 7184-7190.

40. Han A, Lu Y, Zheng Q, Zhang J, Zhao Y, Zhao M, Cui X: Qiliqiangxin Attenuates Cardiac Remodeling via Inhibition of TGF-beta1/Smad3 and NF-kappaB Signaling Pathways in a Rat Model of Myocardial Infarction. Cell Physiol Biochem 2018; 45:1797-1806.

\section{Table}

Table 1 the primers of the target genes

\begin{tabular}{|ll|}
\hline Primers & Sequence \\
\hline FN forward primer & 5'-GGATGCTCCTGCTGTCAC-3' \\
\hline FN reverse primer & 5'-CTGTTTGATCTGGACCTGCAG-3' \\
\hline Col I forward primer & 5'-GGATGCTCCTGCTGTCAC-3' $^{\prime}$ \\
\hline Col I reverse primer & 5'-CTGTTTGATCTGGACCTGCAG-3' $^{\prime}$ \\
\hline GAPDH forward primer & 5'-GTGGATATTGTTGCCATCAATGACC-3' \\
\hline GAPDH forward primer & 5'-GCCCCAGCCTTCTTCATGGTGGT-3' \\
\hline
\end{tabular}

\section{Figures}


A
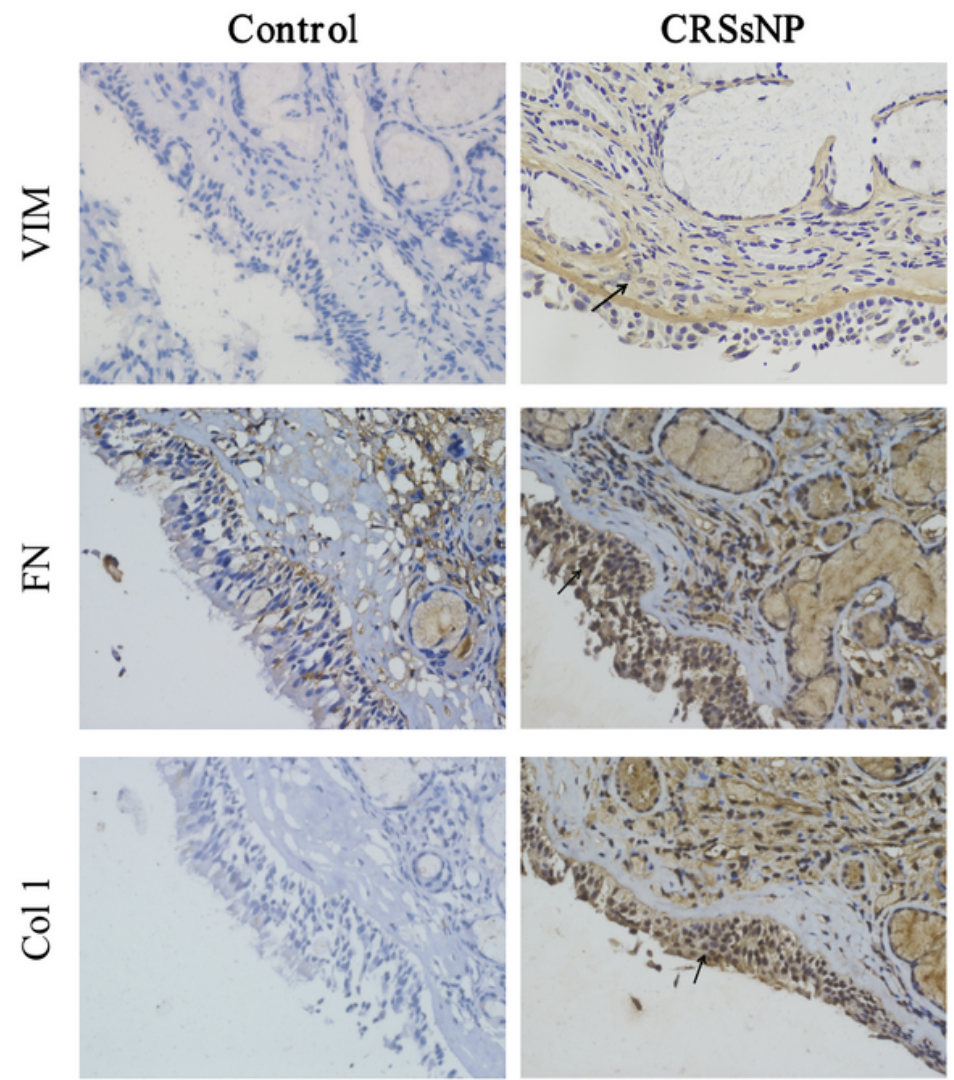

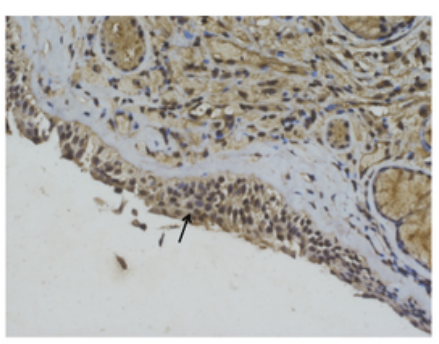

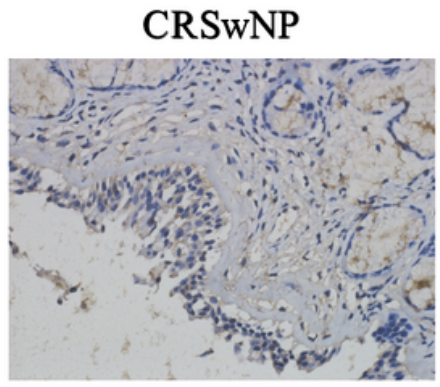
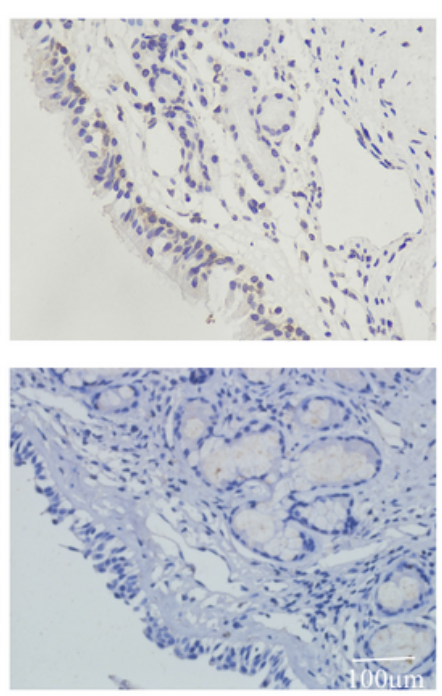

$\mathrm{B}$
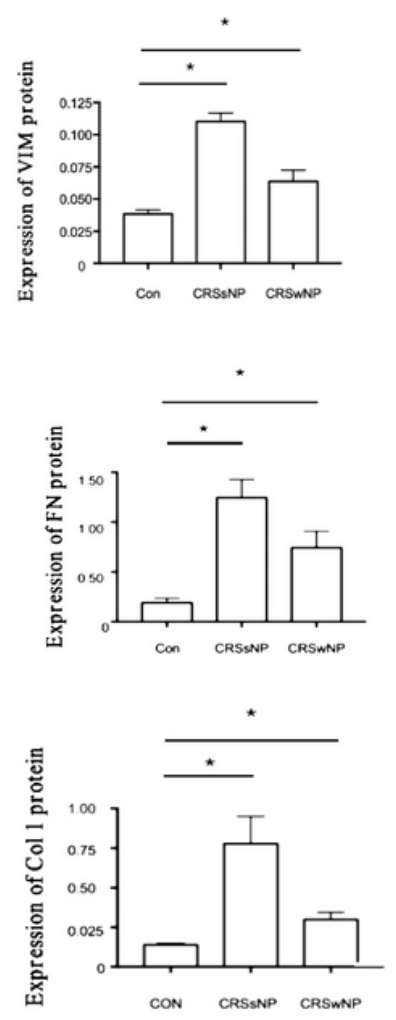

$400 \mathrm{x}$

\section{Figure 1}

The expression of VIM, FN, Col I in Con, CRSsNP and CRSwNP group (A) Immunohistochemical detection of the expression and localization VIM, FN, Col I in the nasal mucosa of Con, CRSsNP and CRSWNP. (B) Statistical analysis of the expression levels of VIM, FN, and Col I in the nasal mucosa of each group. * $\mathrm{p}<$ $0.05,{ }^{*} \mathrm{p}<0.01$, and $* * * \mathrm{p}<0.01$. 


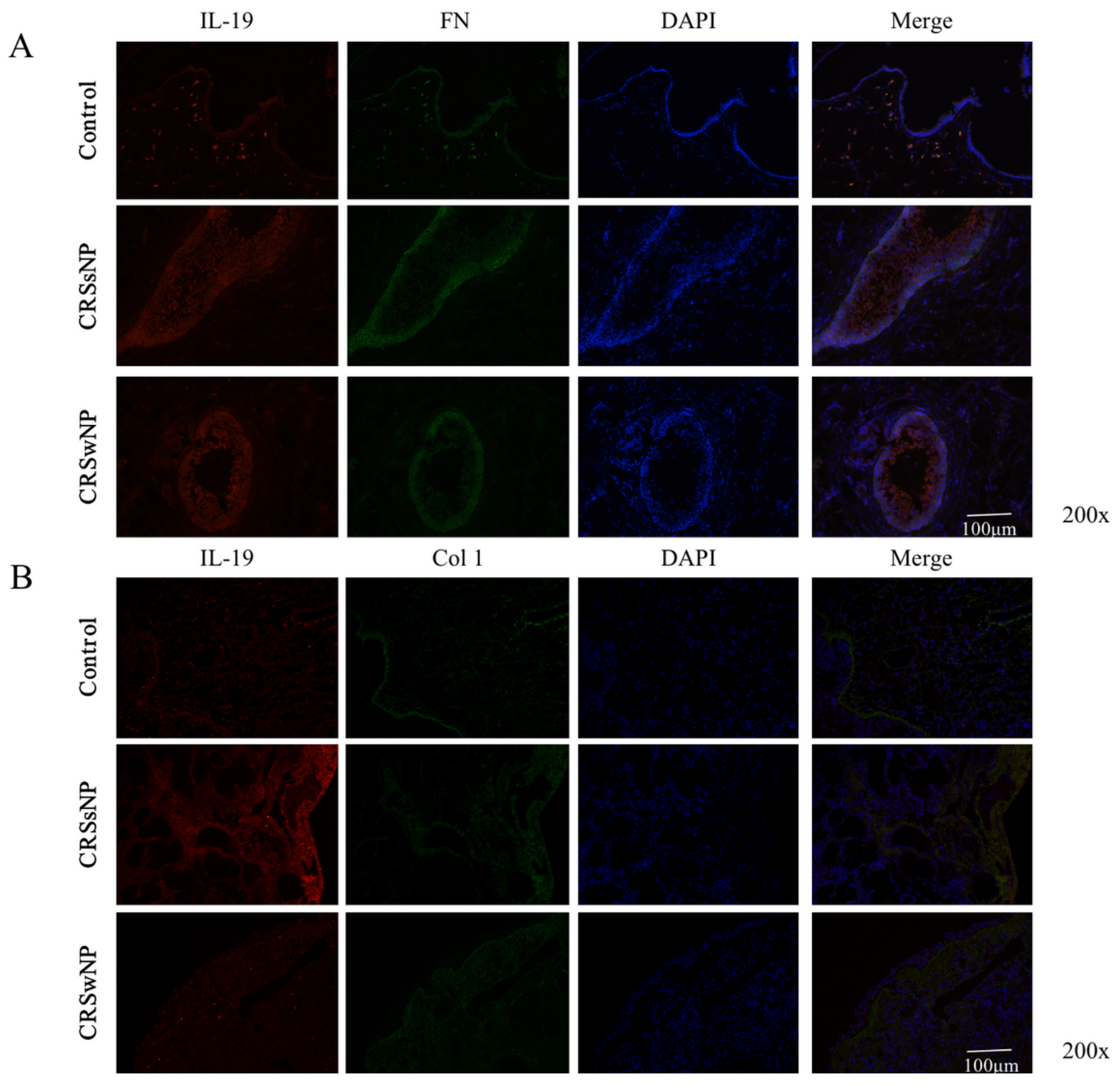

Figure 2

Co-localized expression of IL-19 and FN or Col I in nasal mucosa of CRSsNP. (A) FN and IL-19 in control, CRSsNP and CRSwNP tissues was shown by immunofluorescence double staining at 400x microscopy. (B) Co-localization of IL-19 and Col I expression in control, CRSsNP and CRSwNP tissues was shown by immunofluorescence double staining at 400x microscopy. 
A

IL-19 ng/ml $\quad 0 \quad 50 \quad 100 \quad 200 \quad 300 \quad 400$

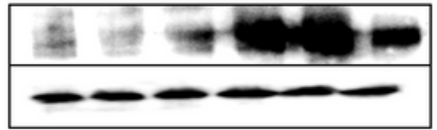

B

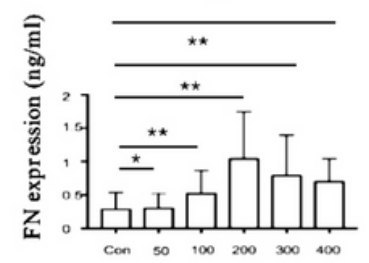

DAPI
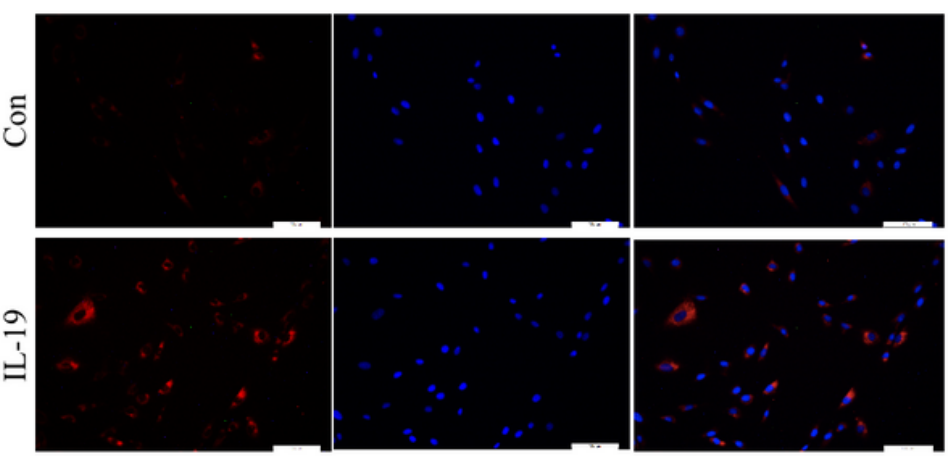

$\mathrm{C}$
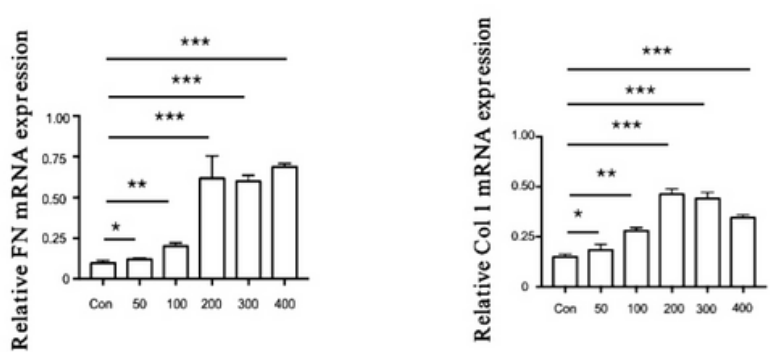

IL-19 ng/ml $\quad 0 \quad 50 \quad 100 \quad 200 \quad 300 \quad 400$
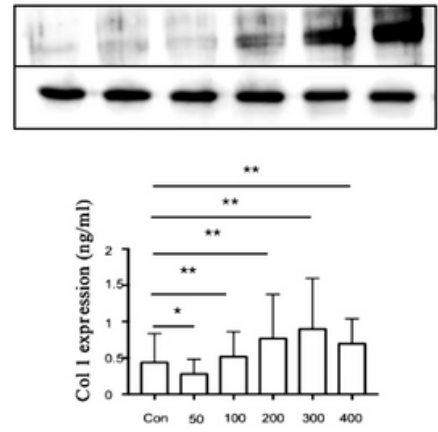

Col 1

DAPI

Merge
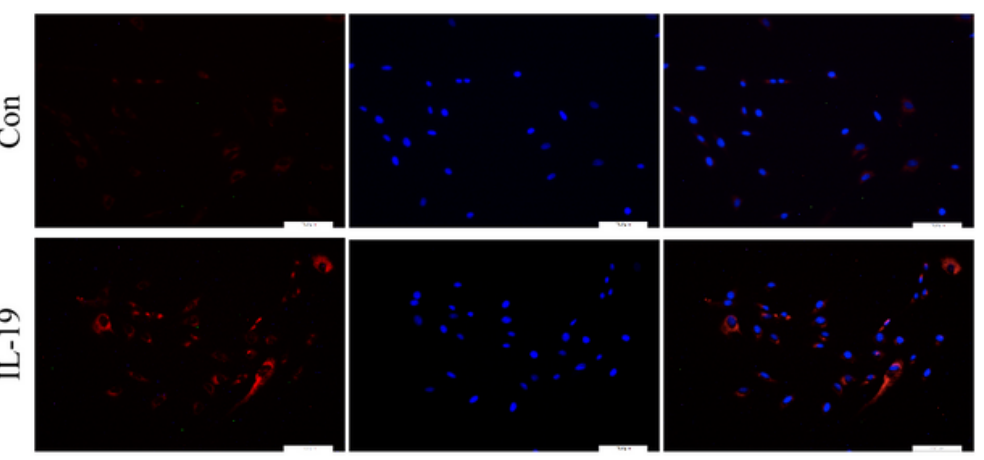

$200 x$

\section{Figure 3}

IL-19 induced FN, Col I expression levels in fibroblasts (A) Fibroblasts were cultured in vitro, and after stimulation with different dose of hIL-19 or not, FN and Col I expression levels were measured by Western blotting. And the quantitative analysis of $\mathrm{FN}$ and $\mathrm{Col} I$ in fibroblasts with different treatment were conducted by using image $\mathrm{J}$ software. (B) FN and Col I expression levels in fibroblasts were observed by immunofluorescence staining under 200x microscope. (C) RT-qPCR was used to determine the mRNA levels of FN and Col I in Fibroblasts with hIL-19 stimulation. ${ }^{*} p<0.05$, ${ }^{*} p<0.01$, and ${ }^{* \star *} p<0.001$. 

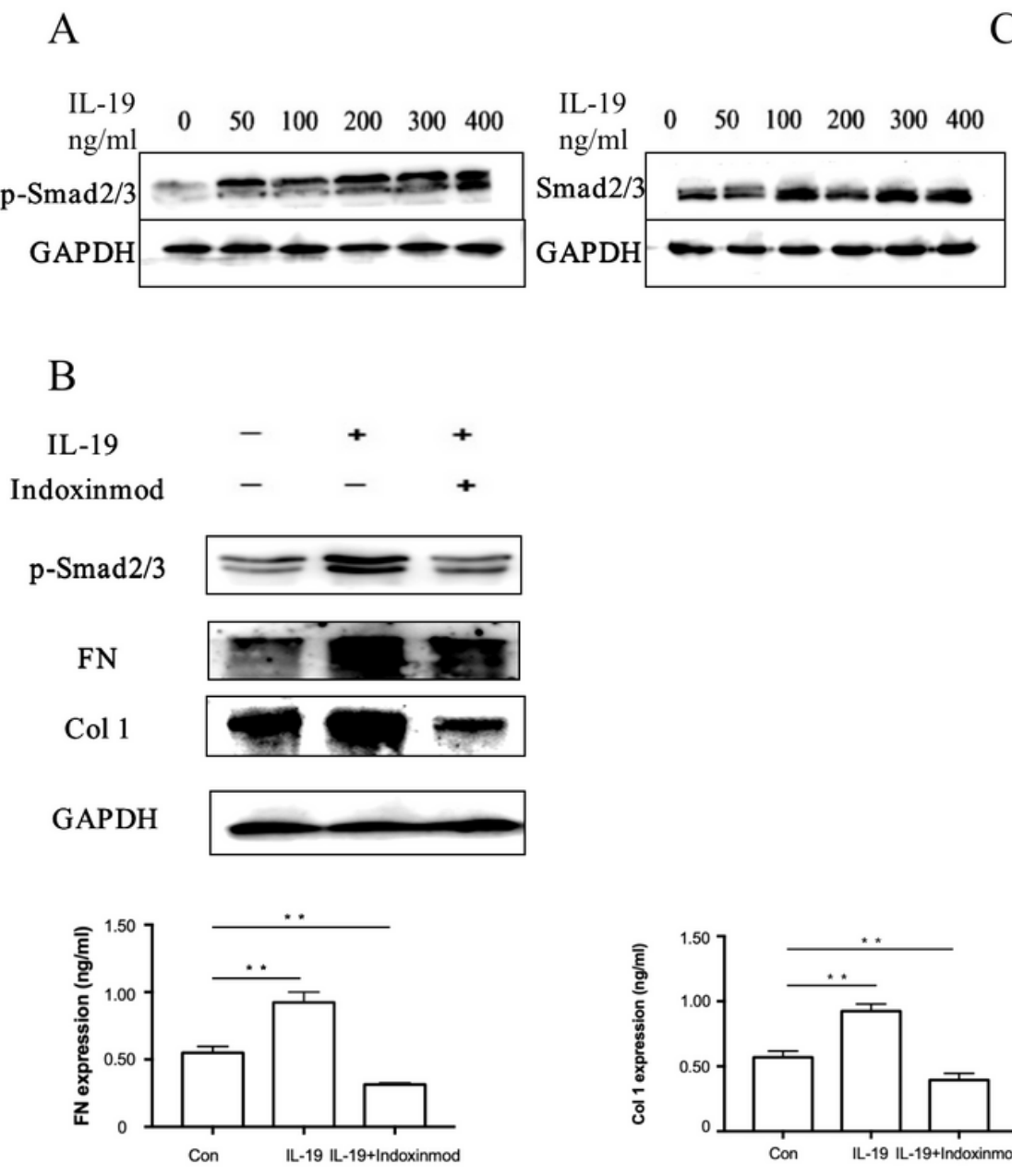

C
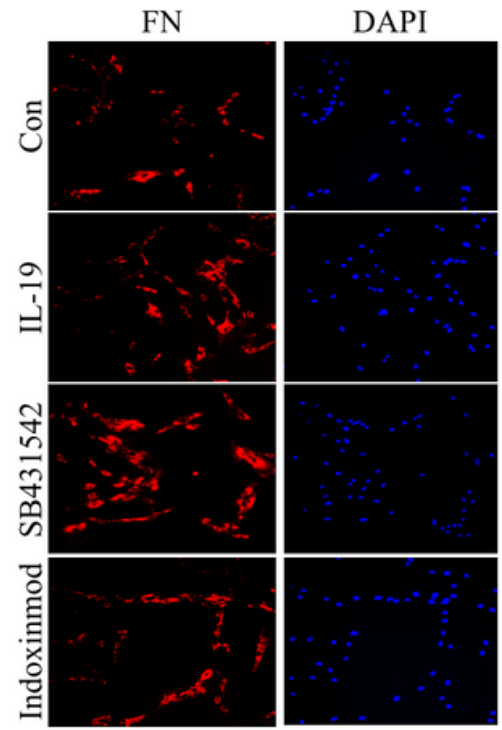

Merge

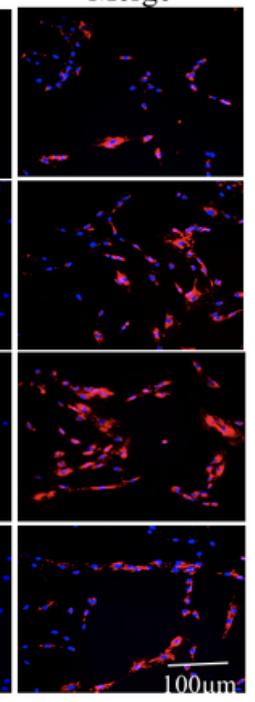

Col 1

DAPI

Merge
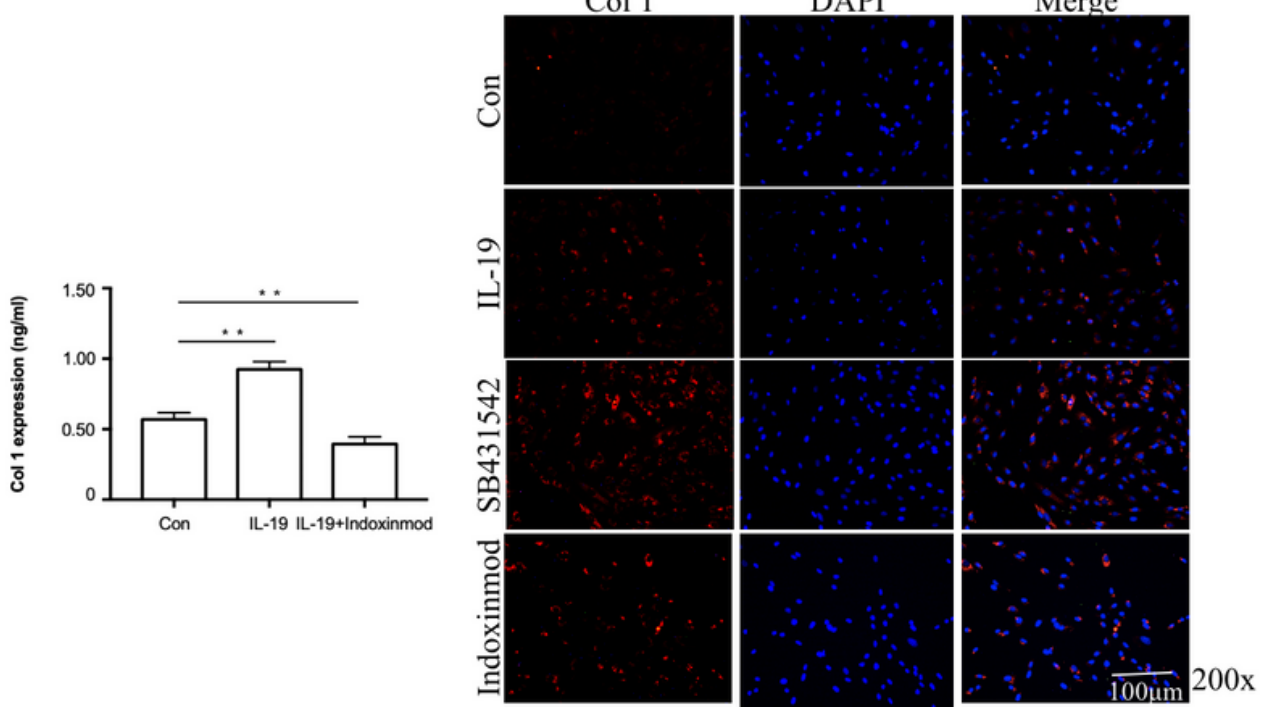

\section{Figure 4}

IL-19 promoted FN, Col I expression in fibroblasts through the Smad2/3 pathway (A) Western blotting was used to detect the activation of Smad2/3 pathway in in fibroblasts with different dose of hIL-19 stimulations. (B) Western blotting to detect the expression levels of pSamd2/3, FN and Col I proteins expression in Fibroblasts with IL-19 stimulation combined with pSmad2/3 pathway inhibitor (Indoxinmod) or not. 10ng/mL Indoxinmod was added 1 hour before $200 \mathrm{ng} / \mathrm{mL}$ hlL-19 treatment. All proteins were harvest 24 hours after hIL-19 treatment. And the quantitative analysis of FN and Col I in fibroblasts with different treatment were conducted by using image $\mathrm{J}$ software. (C) FN and Col I expression levels were observed by using immunofluorescence staining in fibroblasts with different treatments, under 400x microscopy. * $p<0.05$, ** $p<0.01$, and ${ }^{\star *} p<<0.001$. 
A

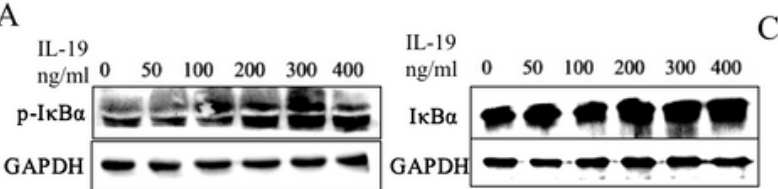

B

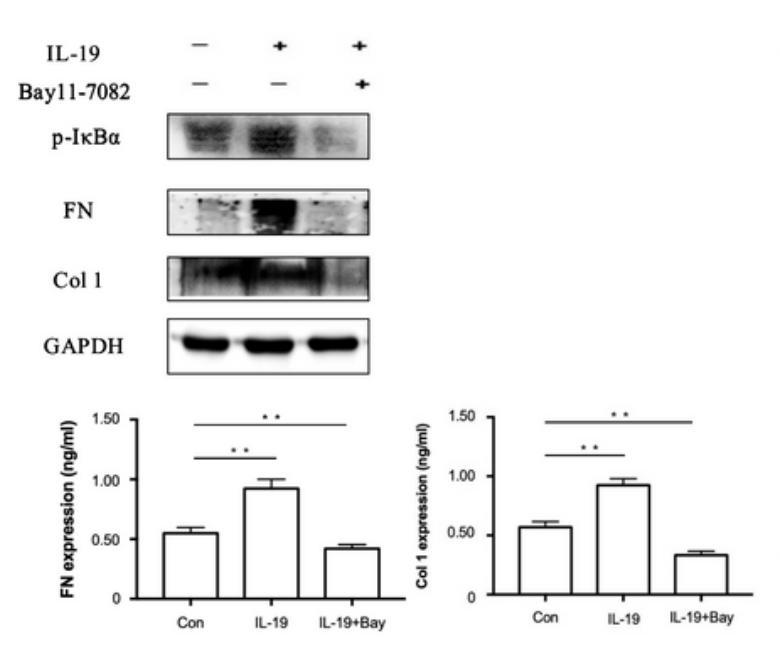

D
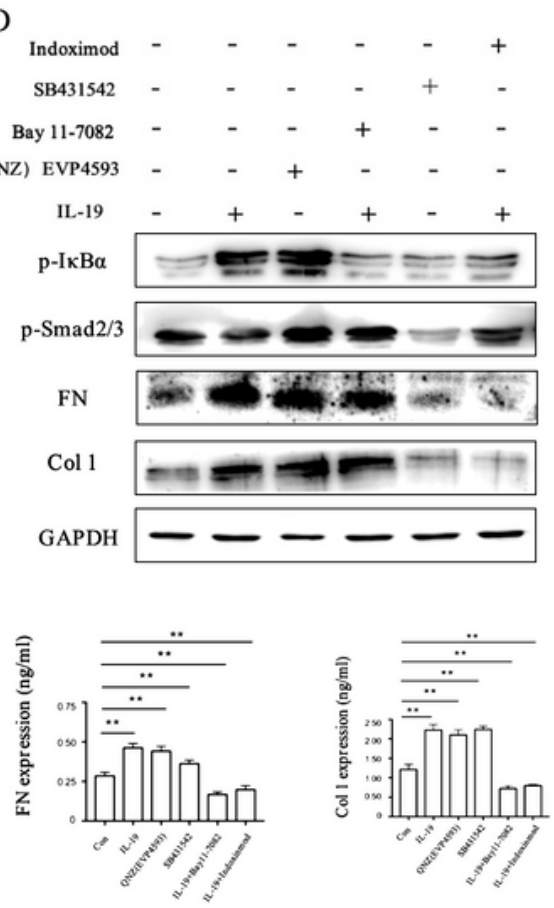

E
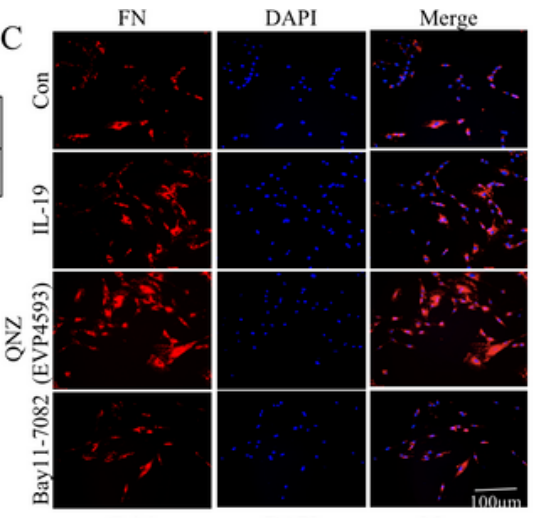

Col 1

DAPI

Merge
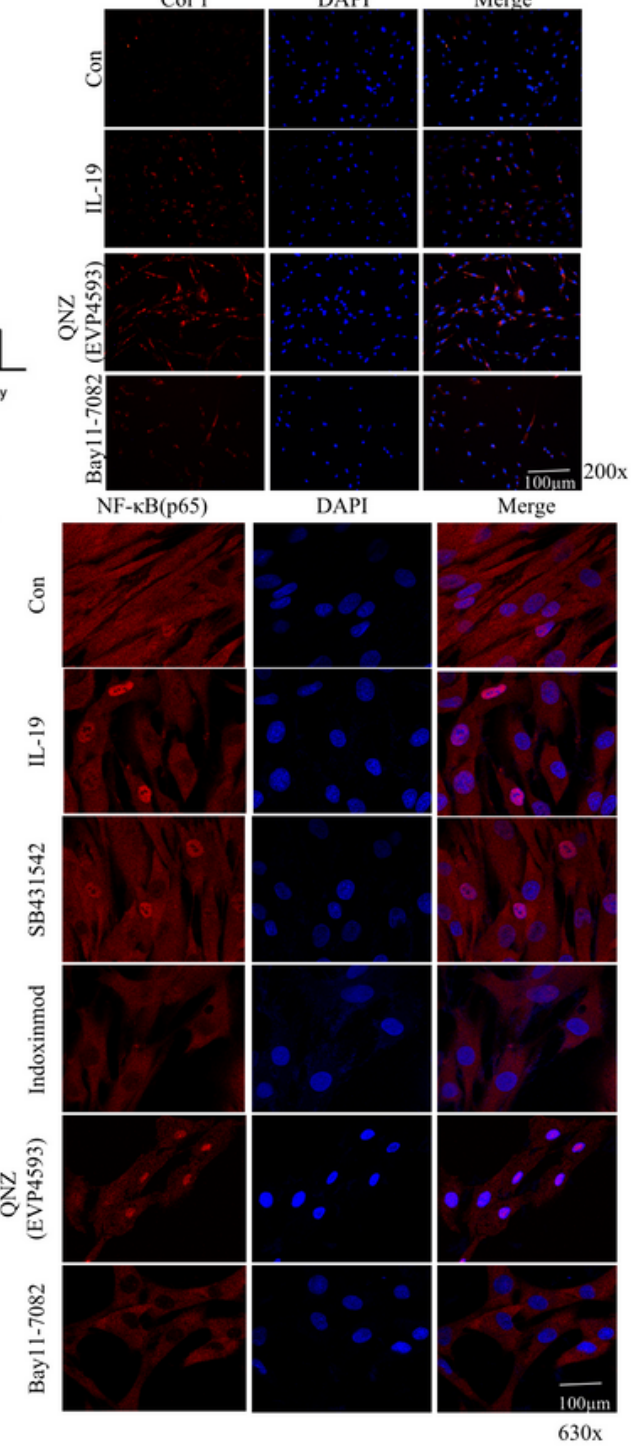

Figure 5

IL-19 promoted FN and Col I expression in fibroblasts via NF-KB-Smad2/3 pathway (A) p-IKBa and IKBa expression levels in fibroblasts with different dose of hIL-19 stimulations were measured by Western blotting. (B) Western blotting was used to detect the expression levels of $\mathrm{p}-\mathrm{IKBa}, \mathrm{FN}$ and $\mathrm{Col} I \mathrm{In}$ in fibroblasts with IL-19 stimulation combined with NF-KB pathway inhibitor (Bay11-7082, Bay) or not. $5 \mathrm{ng} / \mathrm{mL}$ Bay was added 1 hour before 200ng/mL hlL-19 treatment. All proteins were harvest 24 hours 
after hIL-19 treatment. The quantitative analysis of FN and Col I in fibroblasts with different treatment were conducted by using image $\mathrm{J}$ software. (C) The immunofluorescence detection of Col I and FN expression in fibroblasts treated with hIL-19 stimulation combined with $5 \mathrm{ng} / \mathrm{mL}$ NF-KB pathway inhibitor (Bay11-7082), $10 \mathrm{ng} / \mathrm{mL}$ NF-KB pathway activator (QNZ)EVP4593. The Bay and QNZ were added 1 hour before hIL-19 stimulation. (D) Western blotting detection of $\mathrm{p}$-Samd2/3, p-IKBa, FN and Col I expression levels in fibroblasts with hIL-19, NF-KB pathway activator (QNZ) EVP4593, Smad2/3 pathway activator SB431542, and hIL-19 combined with inhibitor Bay11-7082, inhibitor indoximod. The quantitative analysis of $\mathrm{FN}$ and $\mathrm{Col} I$ in fibroblasts with different treatment were conducted by using image $\mathrm{J}$ software. (E) Nuclear transfer of NF-KB(p65) in fibroblasts stimulated with IL-19 as well as NF-KB pathway activator (QNZ) EVP4593, inhibitor Bay11-7082, Smad2/3 pathway activator SB431542, inhibitor indoximod, by using confocal. ${ }^{*} p<0.05,{ }^{*} \mathrm{p}<0.01$, and $* \star \star \mathrm{p}<0.005$. 